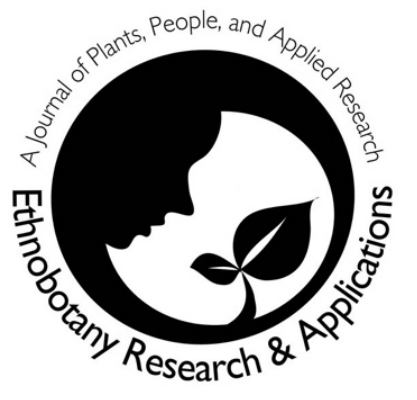

Research

\begin{abstract}
Background: Since appearance of (SARS-CoV-2) in China, December 2019, the pandemic of this human virus is accelerating its spread in Morocco as well as worldwide. The prevention of this disease by strengthening immunity through medicinal plants remains an essential choice of the Moroccan population.
\end{abstract}

Methods: This work consists of a declarative survey by questionnaire divided into two parts: the first concerns the informant and the second concerns the plants used.

Results: Among 267 participants from different regions of Morocco, $67.04 \%$ of respondents use medicinal plants to boost their immunity, disinfect the air, or treat respiratory tract infections that may be related to coronavirus. While $23 \%$ have little confidence in herbal medicine, especially in the face of Covid-19.

Conclusions: The results obtained constitute a valuable source of information to prevent and inhibit the severity of the infection caused by SARS-CoV-2 by strengthening the immune system. The majority of the listed plants are rich in secondary metabolites and essential oils. They are known for their positive biological effects on respiratory functions. These plants may constitute a database for further research to conduct clinical trials to determine the most commonly used plants effectiveness.

Key words: Medicinal plants, prevention, immunity, Covid-19, Morocco

\section{Medicinal plants used to immunity and the intensity of caused by SARS- in Morocco}

\section{Background}

On the $3^{\text {rd }}$ of December, 2020, a group of cases of an epidemic of "pneumonia of unknown cause" in the city health system were discovered. Some cases led to severe complications and death ( $\mathrm{Zu}$ et al. 2020), which triggered a global alert. On March 11, 2020, the World Health Organization (WHO) officially declared the Coronavirus a global pandemic, with 121,000 cases reported. Now, officially it is present in more than 200 countries on five continents (WHO 2020). On July 14th, 2020, the number of Coronavirus infection cases in Morocco was 16,047, with 13,403 recoveries and 259 deaths, which casefatality rate of $1.6 \%$.

Correspondence
Souad Belhaj ${ }^{1 *}$ and Lahcen Zidane
Plant, Animal Productions and Agro-industry
Laboratory, Department of Biology, Faculty of
Sciences, Ibn Tofail University, B.P. 13314000 ,
Kenitra,
Morocco.
*Corresponding Author:
souadbelhaj2050@gmail.com
Ethnobotany Research \& Applications
21:41 (2021)

On July 14th, the Minister of Health said that Morocco had recorded the world's lowest casefatality rate. However, despite the implementation of one of the world's longest confinements, the epidemic has evolved enormously in recent months.

In the absence of a specific cure or vaccine, only care protocols, including antivirals, antibiotics, and drugs are offered to patients. Whereas when patients are in respiratory distress, they are provided by oxygen or placed in intensive care. This therapy is impossible to deliver to most of the population due to 
lack of resources and healthcare structures failure. In this context, the Moroccan population are too insistent on preventive measures to protect against this virus. The majority of the participants confirmed the use of medicinal plants to strengthen their immune systems and to resist the virus attack and defend naturally against bacteria and viruses that seem to associate with SARS-CoV-2. and can often cause serious complications, using natural anti-virals and anti-bacterials.

These locally available natural resources traditionally and spontaneously call upon plants' virtues to treat several ailments since antiquity. Thus, traditional therapeutic practices based on medicinal plants were the only way for Moroccan population to fight against diseases and treat themselves until the beginning of the 20th century (Krati 1991).

The sacred and precious heritage constitutes a response of choice to provide the organism naturally with the necessary bio-active substances known for their positive biological effects on several diseases, such as respiratory and circulatory functions to maintain vital equilibrium (Rejdali 1995).

According to this view, we have conducted a study on local knowledge, attitudes, and practices based on medicinal plants to encounter this pandemic in Morocco.

\section{Material and Methods}

An epidemiological study was conducted in the different prefectures of Morocco: the North, the South, and the Center. This research method consists of a descriptive observational and analytical cross-sectional study to collect information and data on the use of medicinal plants to promote immunity and prevention against the Coronavirus.

Information on the use of medicinal plants was obtained through 311 strictly anonymous questionnaire sheets. They were carried out during the period from May 10 to August 14th. The surveys included the testimonies of the inhabitants of these regions to collect all the information about the citizens and the plants.

These sheets included information about the respondents such as age, gender, occupation, level of academic study, social category, and marital status. In addition to the use of traditional medicinal plants, which allowed us to collect precise information about each plant local name used in phytotherapy against Covid-19, the parts used, the methods of preparation, and the duration of use. Simple random sampling was carried out.
After determining the respondents' vernacular names, we identified the scientific nomenclature using documents concerning vegetation and medicinal plants in Morocco, such as Fennane et al. 2007. We consulted botanical databases such as (https://wwwBotanique. Org/systematique-vegetaleclassification-plantes-article24342/).

Moreover, in order to evaluate the socio-cultural importance of plants used to promote immunity and prevention of Coronavirus, we used different analytical tools, for example, Citation frequency (FC), relative citation frequency (RFC), use value by species (VUe), importance value (VI), Prevalence estimate $(P)$, and Prevalence ratio (RP). The data collected and recorded on the survey forms were then entered and statistically analyzed using SPSS Statistics (Social Science Statistical Software, Version 21) and Excel 2010.

Use value by species (VUe): This formula was calculated according to the method used by Houmenou et al. (2017). It represents the number of times the species are cited in recipes.

The value of importance ( $\mathrm{VI})$ : Is the ratio of the number of times the species is cited in the recipes (VUe) by all listed species (N).

$$
\text { VI = VUe / N (Houmenou et al. 2017). }
$$

Relative Citation Frequency (RCF): It is the response rate of the organs used by the type of species. It can be applied in ethnobotany to calculate the value of biological plant taxon.

The RFC is expressed using:

RFC= FC / NS x100 (Dossou et al. 2012).

FC: it refers to the number of respondents who gave a positive response to the plants use for a given pathology, while $\mathrm{N}$ : is the total number of people interviewed. Its value ranges from 0 to 1 and indicates the most used organs for each type in the environment. The value 0 indicates that the organ is not used and 1 when all respondents are using it.

Estimated disease prevalence for an exposed group: it refers to the cases affected by the study population epidemic.

The mathematical formula is: $\mathrm{P} 1=\mathrm{a} / \mathrm{E} 1 \times 100$

Where; a: indicates the number of cases exposed to Covid19 during a given period, and E1: the total number of interviewees (Feingold 1998).

Prevalence ratio: $\mathrm{RP}=\mathrm{P} 1 / \mathrm{P0}$ (Feingold, 1998). 


\section{Results and Discussion}

During this study, the total number of contacted respondents was 311. From this number, 267 agreed to respond to our questionnaire. One hundred seventy-nine respondents, or $67.04 \%$, used traditional medicine at the beginning of the epidemic to fight against the Coronavirus. These respondents live in the different regions of Morocco from North to South. $39.56 \%$ live in Casablanca Settat, $27.31 \%$ in Fes Meknes, $19.13 \%$ in Draa-Tafilalet, $11.4 \%$ in Tanger-Tetouan-Al Hoceima, and 6.6\% in Guelmim Oued Noun. We sent to these participants the link to a strictly anonymous self-questionnaire.

Women $(72.06 \%)$, especially those who are married (56.98\%) used more medicinal plants for prevention and controlling SARS-CoV-2. This type of therapy occupies an essential place in the daily lives of Moroccan women. The results were consistent with other studies conducted in several different Moroccan regions (Belhaj et al. 2020; Chaachouay et al. 2019) and Africa (Ambe et al. 2015; Ndjouondo et al. 2015; Blama \& Mamine 2013) on the use of medicinal plants. The results can be explained by the primordial role of women in conserving the therapeutic traditions based on medicinal plants used to control and prevent diseases in their homes. Thus, women are rigorously involved in the search for the family's livelihood.

The respondents were composed of $68.71 \%$ adults, $14 \%$ youth, and $6.14 \%$ elders. Adults were much more represented than the others (Table 1).

Table 1. Demographic profile of informants interviewed

\begin{tabular}{|c|c|c|c|}
\hline Variables & Categories & Total & Percentage(\%) \\
\hline \multirow{4}{*}{ Gender } & Female & 129 & 72.06 \\
\cline { 2 - 4 } & Male & 50 & 27.93 \\
\hline \multirow{4}{*}{ Age groups } & $19-30$ years & 45 & 25.14 \\
\cline { 2 - 4 } & $30-50$ years & 123 & 68.71 \\
\cline { 2 - 4 } & $50-70$ years & 11 & 6.14 \\
\hline Family situation & Married & 102 & 56.98 \\
\cline { 2 - 4 } & Single & 77 & 43.02 \\
\hline \multirow{4}{*}{$\begin{array}{c}\text { Occupational } \\
\text { categories }\end{array}$} & Primary & 9 & 5.03 \\
\cline { 2 - 4 } & Secondary & 57 & 31.84 \\
\cline { 2 - 4 } & University & 113 & 63.13 \\
\cline { 2 - 4 } & Employees & 61 & $34.08 \%$ \\
\cline { 2 - 4 } & Traders & 32 & $17.88 \%$ \\
\cline { 2 - 4 } & Entrepreneurs & 11 & $6.14 \%$ \\
\cline { 2 - 4 } & Students & 21 & $11.73 \%$ \\
\cline { 2 - 4 } & Retired & 47 & $26.26 \%$ \\
\hline
\end{tabular}

The use of medicinal plants varied according to academic level. The majority of them had university level $(63.13 \%)$, and $31.84 \%$ has a high school level. Only $5.03 \%$ had only elementary school level (Table 1). These results diverge from almost all studies on the use of medicinal plants in Morocco (Fouad \& Lahcen 2020, Briguiche \& Zidane 2019, Rhattas et al. 2016). This difference was due to the confinement and social distancing measures imposed, which forced us to use the online questionnaires. The population study income was very diversified: $34.08 \%$ are salaried employees, $26.26 \%$ are retired, $17.88 \%$ are shopkeepers, $11.73 \%$ are students, $6.14 \%$ are entrepreneurs $3.91 \%$ are unemployed (Table 1). The findings of several ethnobotanical studies in different regions of Morocco were linked to the users of medical plants. With higher education level, the use of phytotherapy decreased (Belhaj et al. 2020, Idm'hand et al. 2019, Benlamdini et al. 2014). In relation to the financial situation of the respondents, we noticed an increase of the use of medicinal plants related to the decrease in the monthly income (Chaachouay et al. 2019, Al-Hadri 2019). This big difference with the results of our present study would come from the fact that the participants sought to prevent against this pandemic either by hygiene measures or by strengthening the immune system, especially when the speed, spread and contamination by this virus were rapid, and the access to modern health care was difficult. This situation made the use of plants and natural products available a first line of defense in order to improve the antiviral immune system, and consequently keep the body more resistant to viral infections. These plants are revealed a variety of secondary metabolites and bioactive substances, which most of them are known for their antioxidant, antimicrobial, anti-inflammatory and even antiviral effects. (Table 3). 
Table 2. Inventory of plant species used to boost immunity and decrease the intensity of infection caused by Covid-19.

\begin{tabular}{|c|c|c|c|c|c|c|c|c|}
\hline Families & Scientific name & Local name & Parts used & Preparation & FC & RFC & VUe & VI \\
\hline \multicolumn{9}{|c|}{ Medicinal plants used to promote immunity } \\
\hline Amaryllidaceae & Allium sativum L. & Touma & Bulbs & Cooked & 97 & 0.54 & 41 & 1.64 \\
\hline Apiaceae & Carum carvi $\mathrm{L}$. & Karwiya & Fruit & Powder & 56 & 0.31 & 13 & 0.52 \\
\hline Araliaceae & Panax ginseng C.A. Mey & Ginsing & Rhizome & Decoction, Powder & 69 & 0.38 & 23 & 0.92 \\
\hline \multirow{2}{*}{ Asteraceae } & Artemisia herba alba Asso & Chih & Aerial parts & Maceration & 41 & 0.23 & 19 & 0.76 \\
\hline & Matricaria chamomilla L. & Lbabounj & Flowers & Infusion & 54 & 0.3 & 12 & 0.48 \\
\hline Brassicaceae & Lepidium sativum $\mathrm{L}$. & Habrchad & Seeds & Decoction & 71 & 0.4 & 26 & 1.04 \\
\hline Capparaceae & Capparis spinosa L. & Lkabbar & Fruits & Decoction & 68 & 0.38 & 21 & 0.84 \\
\hline Cucurbitaceae & Cucurbita maxima L. & Lgraa hamra & Seeds & Powder & 33 & 0.18 & 16 & 0.64 \\
\hline Cupressaceae & Juniperus communis L. & Larâar & Leaves & Infusion & 49 & 0.27 & 21 & 0.84 \\
\hline \multirow{2}{*}{ Fabaceae } & Glycyrrhiza glabra L. & Aarqssuss & Roots & Decoction & 14 & 0.07 & 6 & 0.24 \\
\hline & Trigonella foenum-graecum $\mathrm{L}$. & Halba & Seeds & Powder & 63 & 0.35 & 21 & 0.84 \\
\hline Iridaceae & Crocus sativus $\mathrm{L}$. & Za' farān & Stigmas & Decoction & 89 & 0.5 & 32 & 1.28 \\
\hline Lamiaceae & Salvia officinalis L. & Salmiya & Leaves & Decoction & 72 & 0.4 & 28 & 1.12 \\
\hline Lamiaceae & Ocimum Basilicum L. & Lhbaq & Aerial parts & Infusion & 15 & 0.08 & 11 & 0.44 \\
\hline Lauraceae & Cinnamomum verum J. Presl & El qarfa & Barks & Maceration & 42 & 0.23 & 23 & 0.92 \\
\hline Moraceae & Ficus carica $L$. & Karmouss & Fruits & Evaporation & 69 & 0.38 & 27 & 1.08 \\
\hline Oleaceae & Olea europaea L. & Zaytoun & Leaves & Decoction & 102 & 0.57 & 58 & 2.32 \\
\hline Piperaceae & Piper nigrum L. & Ibzar & Seeds & Powder & 29 & 0.16 & 15 & 0.6 \\
\hline Poaceae & Hordeum vulgare $\mathrm{L}$. & Chaâir & Seeds & Maceration & 77 & 0.43 & 31 & 1.24 \\
\hline Portulacaceae & Portulaca oleraceae L. & Rejla & Aerial parts & Cooked & 43 & 0.24 & 18 & 0.72 \\
\hline Renonculaceae & Nigella damascena L. & Assanouj & Seeds & Powder & 66 & 0.37 & 18 & 0.72 \\
\hline Theaceae & Camellia sinensis L. & Atay & Leaves & Decoction & 43 & 0.24 & 22 & 0.88 \\
\hline Vitaceae & Vitis vinifera L. & Laanab & Leaves & Infusion & 99 & 0.55 & 49 & 1.96 \\
\hline Zingiberaceae & Curcuma longa L. & Kherqum & Roots & Powder & 81 & 0.45 & 37 & 1.48 \\
\hline Zingiberaceae & Zingiber officinale Roscoe & Zanjabil & Roots & Decoction & 106 & 0.59 & 53 & 2.12 \\
\hline \multicolumn{9}{|c|}{ Medicinal plants used to purify the air } \\
\hline Anacardiaceae & Pistacia atlantica Desf & Lbtam & Barks & Fumigation & 35 & 0.19 & 17 & 0.68 \\
\hline Asteraceae & Artemisia herba alba Asso & Chih & Aerial parts & Inhalation & 83 & 0.46 & 31 & 1.24 \\
\hline Cupressaceae & Juniperus phoenicea L. & L arâar, El-horr & Aerial parts & Fumigation & 78 & 0.43 & 29 & 1.16 \\
\hline Juglandaceae & Juglans regia L. & Lgargaie & Barks & Inhalation & 14 & 0.07 & 18 & 0.72 \\
\hline \multirow{3}{*}{ Lamiaceae } & Lavandula dentata L. & Lkhzama & Aerial parts & Inhalation & 91 & 0.5 & 48 & 1.92 \\
\hline & Mentha pulegium L. & Fliyyou & Aerial parts & Inhalation & 78 & 0.43 & 51 & 2.04 \\
\hline & Origanum compactum Benth & Zaater & Aerial parts & Inhalation & 39 & 0.22 & 21 & 0.84 \\
\hline
\end{tabular}




\begin{tabular}{|c|c|c|c|c|c|c|c|c|}
\hline Lythraceae & Punica granatum L. & Rommane & Fruit bark & Fumigation & 17 & 0.09 & 9 & 0.36 \\
\hline \multirow{2}{*}{ Myrtaceae } & Eucalyptus globulus Labill & Eucalyptus & Leaves & Inhalation & 91 & 0.5 & 59 & 2.36 \\
\hline & Eugenia caryophyllata Thunb & Qronfel & Cloves & Fumigation & 89 & 0.5 & 67 & 2.68 \\
\hline Nitrariaceae & Peganum harmala L. & Harmel & Seeds & Fumigation & 86 & 0.48 & 44 & 1.76 \\
\hline \multicolumn{9}{|c|}{ Medicinal plants used to alleviate some symptoms caused by the respiratory infection due to the Coronavirus } \\
\hline Amaryllidaceae & Allium cepa L. & Lbassala & Fruit & Relievs cough and fever & 42 & 0.23 & 29 & 1.16 \\
\hline Anacardiaceae & Pistacia atlantica Desf. & Lbtam & Barks & Soothes headaches & 37 & 0.2 & 22 & 0.88 \\
\hline \multirow[t]{2}{*}{ Apiaceae } & Carum carvi L. & Karwiya & Fruit & $\begin{array}{l}\text { Relieves cough and } \\
\text { decrease tiredness }\end{array}$ & 56 & 0.31 & 17 & 0.68 \\
\hline & Smyrnium olusatrum L. & Lhayar & Leaves & Relieves asthma attacks & 54 & 0.3 & 29 & 1.16 \\
\hline Apocynaceae & Caralluma europaea (Guss.) & Darmouss & Fruit & Relieves asthma attacks & 33 & 0.18 & 12 & 0.48 \\
\hline Araliaceae & Hedera helix L. & Lwwaya & Leaves & Relieves cough & 18 & 0.1 & 9 & 0.48 \\
\hline Asteraceae & Artemisia absinthium L. & Chiba & Aerial parts & Relieves fever & 39 & 0.22 & 21 & 0.84 \\
\hline Brassicaceae & Brassica rapa $L$. & Left & Roots & Relieves cough & 55 & 0.3 & 31 & 1.24 \\
\hline Caprifoliaceae & Sambucus nigra L. & Sembouqa & Leaves & Relieves fever & 41 & 0.22 & 11 & 0.44 \\
\hline Ericaceae & Arbutus unedo L. & Barnnou & Leaves & Decrease tiredness & 45 & 0.25 & 22 & 0.88 \\
\hline \multirow{2}{*}{ Fabaceae } & Ceratonia siliqua L. & Lkharoub & Leaves & Decrease tiredness & 41 & 0.23 & 34 & 1.36 \\
\hline & Trigonella foenum-graecum L. & Halba & Seeds & Relieves cough & 77 & 0.43 & 41 & 1.64 \\
\hline \multirow[b]{2}{*}{ Myrtaceae } & Eucalyptus globulus Labil & Eucalyptus & Barks & Relieves cough & 82 & 0.46 & 59 & 2.36 \\
\hline & Eugenia caryophyllata Thunb & Qronfel & Cloves & $\begin{array}{l}\text { Relieves cough and } \\
\text { soothes head aches }\end{array}$ & 39 & 0.22 & 37 & 1.48 \\
\hline Pinaceae & Cedrus atlantica Manetti ex Endl. & Arz & Bark & $\begin{array}{l}\text { Fights coughs and } \\
\text { bronchitis }\end{array}$ & 32 & 0.18 & 21 & 0.84 \\
\hline Plantaginaceae & Plantago major L. & Lmassas & Aerial parts & Relieves asthma attacks & 29 & 0.16 & 12 & 0.48 \\
\hline Salicaceae & Populus alba L. & Safsaf & Leaves & Relieves asthma attacks & 41 & 0.23 & 23 & 0.92 \\
\hline Verbenaceae & Aloysia triphylla (L'Herit.) Britton & Louiza & Leaves & Decrease tiredness & 61 & 0.34 & 38 & 1.52 \\
\hline \multicolumn{3}{|c|}{$P 1=11.21 \%$} & \multicolumn{6}{|c|}{$\mathrm{P} 0=88.79 \%$} \\
\hline
\end{tabular}


Table 3. The biological activities and main active components of medicinal plants used to strengthen immunity and decrease the intensity of infection caused by Covid-19 in Morocco

\begin{tabular}{|c|c|c|c|}
\hline Familiy & Scientific name & Identified key active components & Biological properties \\
\hline \multirow[t]{2}{*}{ Amaryllidaceae } & Allium cepa L. & $\begin{array}{l}\text { Diallyl disulfide, Diallyl trisulfide, Allyl methyl trisulfide, } \\
\text { Diallyl sulfide, Diallyl tetrasulfide, Allyl methyl disulfide } \\
\text { (Mnayer et al. } 2014 \text { ). }\end{array}$ & $\begin{array}{l}\text { Antimicrobial, Antibacterial, Antioxidant (Mnayer et } \\
\text { al. } 2014 \text { ). }\end{array}$ \\
\hline & Allium sativum $\mathrm{L}$. & $\begin{array}{l}\text { Allyltrisulfide, Diallyl disulfide, Flavonoids (Sasaki et } \\
\text { al. 2007) }\end{array}$ & Antibacterial, Anti-hemolytic (Nezla 2018) \\
\hline Anacardiaceae & Pistacia atlantica Desf & $\begin{array}{l}\text { Monoterpene hydrocarbons, } \alpha \text {-Pinene, } \beta \text {-Pinene, } \\
\text { Oxygenated monoterpenes, Bornyl acetate, } \\
\text { Monoterpenes, Sesquiterpenes, } \\
\text { Terpinen-4-ol, Elemol (Barrero et al. 2005) }\end{array}$ & $\begin{array}{l}\text { Antioxidant, Antimicrobial, Antifungal } \\
\text { (Benhammou et al. 2008) }\end{array}$ \\
\hline Apocynaceae & Caralluma europaea Guss. & $\begin{array}{l}\beta \text { Carotene, Terpinolene, } \alpha \text { Terpinene, linalool, } \\
\text { hexadecanoïc acid , } \beta \text { Pinene, } \beta \text { Eudesmol (Dra et al. } \\
\text { 2018) }\end{array}$ & $\begin{array}{l}\text { Antimicrobial, Antibacterial, Antioxidant (Dra et al. } \\
\text { 2018) }\end{array}$ \\
\hline \multirow[t]{2}{*}{ Apiaceae } & Carum carvi L. & $\begin{array}{l}\text { Terpenes, Phenylpropanoides } \\
\text { Flavonoids, Coumarines, Tannins, Quinones (El Kolli } \\
\text { 2018) }\end{array}$ & $\begin{array}{l}\text { Antioxidant, Antibacterial (Thippeswamy et al. } \\
\text { 2013) } \\
\text { Anti-hyperglycaemic (Eddouks et al. 2004) }\end{array}$ \\
\hline & Smyrnium olusatrum L. & $\begin{array}{l}\text { Sabinene, Curzerene, Methyl-1-Benzyl-2-, } \\
\text { Oxocyclooctane Carboxylate, } \alpha-P i n e n e, \text { Cryptone, } \beta- \\
\text { Pinene (Daroui-Mokaddem et al. 2010) }\end{array}$ & $\begin{array}{l}\text { Antiproliferative, Antimicrobial, Antioxidant } \\
\text { (Quassinti et al. 2013) }\end{array}$ \\
\hline \multirow[t]{2}{*}{ Araliaceae } & Hedera helix L. & $\begin{array}{l}\text { Unsaturated sterols, Tannins, Phenolic compounds, } \\
\text { Terpenoids, Glycosides, Alkaloids, Flavonoids, } \\
\text { Carbohydrates, Reducing sugars, Saponins, } \\
\text { Vitamins, Minerals (Al-Snafi. 2018) }\end{array}$ & $\begin{array}{l}\text { Anti-inflammatory, Analgesic, Immunological, } \\
\text { Anticancer, Antimutagenic, Antimicrobial, Anti- } \\
\text { Parasitic, Gastrointestinal, Antithrombin, } \\
\text { Respiratory activity (Al-Snafi. 2018) }\end{array}$ \\
\hline & Panax ginseng C.A. Mey & Ginsenosides, Saponins (Goetz 2004) & $\begin{array}{l}\text { Anti-septicaemic (Lim et al. 2002) } \\
\text { Anticancer, Cardiovascular (Goetz 2004) }\end{array}$ \\
\hline \multirow[t]{3}{*}{ Asteraceae } & Artemisia absinthium L. & $\begin{array}{l}\text { Saponins, Phytosterols, Carbohydrates, Proteins, } \\
\text { Tannin, Phenolic compounds, Amino acid, Flavonoid } \\
\text { (Ashok \& Upadhyaya 2013) }\end{array}$ & $\begin{array}{l}\text { Analgesic, Anti-inflammatory, Antimicrobial, } \\
\text { Antioxidants, Hepatoprotective activity (Ashok \& } \\
\text { Upadhyaya 2013) }\end{array}$ \\
\hline & Artemisia herba alba Asso & $\begin{array}{l}\text { Flavonoids (Qnais et al. 2014) } \\
\text { Davanone, Caryophyllene oxide, Heptadecatrien-1-ol, } \\
\text { Spathulenol, a Camphole aldehyde } \\
\text { (Moumni et al. 2013) }\end{array}$ & $\begin{array}{l}\text { Analgesic, Anti-inflammatory (Qnais et al. 2014) } \\
\text { Antibactériennes, Antiradicalaires (Akrout et al. } \\
\text { 2010) }\end{array}$ \\
\hline & Matricaria chamomilla L. & $\begin{array}{l}\text { Germacene D, Bicyclogermacrene, } \beta \text {-Farnesene, } \alpha \text { - } \\
\text { Bisabolol oxide B, } \alpha \text {-Bisabololo, Chamazulene, } \alpha \text { - }\end{array}$ & Hepatoprotective (Tavakol et al. 2015) \\
\hline
\end{tabular}




\begin{tabular}{|c|c|c|c|}
\hline & & $\begin{array}{l}\text { Bisabolol oxide A, Guaizulene, Cis-z- } \alpha \text {-Bisabolene } \\
\text { expoxide, Cis-ene-yne-Dicycloether, Trans-ene-yne- } \\
\text { Dicycloethe (Ali 2013) }\end{array}$ & $\begin{array}{l}\text { Antifungal, Antiaflatoxigenic, Antioxidant, } \\
\text { Anticancer (Ali 2013) }\end{array}$ \\
\hline \multirow[t]{2}{*}{ Brassicaceae } & Brassica rapa $L$. & $\begin{array}{l}\text { Glucosinolates, Polyphenols, Amino acids, Organic } \\
\text { acids, Sugars, Sugar alcohols, Carotenoids, Vitamins } \\
\text { (Šamec et al. 2017) }\end{array}$ & $\begin{array}{l}\text { Anticancer, Anti-inflammatory, Antioxidants, } \\
\text { Antiobesity, hypolipidemic, hypoglycemic, } \\
\text { Gastrointestinal activity (Šamec et al. 2017) }\end{array}$ \\
\hline & Lepidium sativum L. & $\begin{array}{l}\text { Alkaloids, Tannins, Saponins, Anthraquinones } \\
\text { (Rehman et al. 2012) }\end{array}$ & $\begin{array}{l}\text { Antidiarrheal, Antispasmodic (Rehman et al. 2012) } \\
\text { Antimicrobial, Antioxidant (Belkhiri 2018) }\end{array}$ \\
\hline Capparaceae & Capparis spinosa L. & $\begin{array}{l}\text { Alkaloids, Flavonoids, Anthocyanins, } \\
\text { Leucoanthocyanes, Catechols, Tannins, } \\
\text { Saponosides, Sterols, Triterpenes, Anthraquinones, } \\
\text { Reducing compounds, Mucilages, Oses, Holosides, } \\
\text { Tetrahydrocannabinols (Fadili et al. 2017) }\end{array}$ & $\begin{array}{l}\text { Antiproliferative, Antifungal (Lam \& Ng 2009) } \\
\text { Antioxidant (Fadili et al. 2017) }\end{array}$ \\
\hline Cucurbitaceae & Cucurbita maxima L. & $\begin{array}{l}\text { Carotenoids, Polyalcohol, Sterols, Protochlorophyll } \\
\text { (Ghedira \& Goetz 2013) }\end{array}$ & $\begin{array}{l}\text { Antihelminthic, Antischistosomial, Anti- } \\
\text { inflammatory, Antiandrogenic (Ghedira \& Goetz } \\
\text { 2013) }\end{array}$ \\
\hline \multirow[t]{2}{*}{ Cupressaceae } & Juniperus communis L. & $\begin{array}{l}\text { Monoterpenes:Sabinene, Limonene, } \alpha-P i n e n, \\
\text { Terpinene-4-ol. (Mansouri et al. 2011) } \\
\text { Terpinyl acetate, Germacrene (Marongiu et al. 2017) }\end{array}$ & $\begin{array}{l}\text { Antiviral, Antibacterial, Antiproliferative, } \\
\text { Antimycotic } \\
\text { (Marongiu et al. 2017) }\end{array}$ \\
\hline & Juniperus phoenicea L. & $\begin{array}{l}\text { a-Pinene, Sabinene, } \alpha \text {-Cedrol, Monoterpene } \\
\text { Hydrocarbon, Oxygenated sesquiterpenes (El-Sawi et } \\
\text { al .2007) }\end{array}$ & $\begin{array}{l}\text { Cytotoxic, Antioxidant (El-Sawi et al .2007). } \\
\text { Antibacterial (Drider \& Kada 2019) }\end{array}$ \\
\hline Ericaceae & Arbutus unedo L. & $\begin{array}{l}\text { Phenolic acids, Flavonoids, Tannins, Anthocyanins, } \\
\text { Fatty acids, Vitamins, Organic acids, Volatiles, } \\
\text { Minerals (Miguel et al. 2014) }\end{array}$ & $\begin{array}{l}\text { Anti-inflammatory, Antibacterial, Antioxidant } \\
\text { (Miguel et al. 2014) }\end{array}$ \\
\hline \multirow[t]{3}{*}{ Fabaceae } & Ceratonia siliqua L. & $\begin{array}{l}\text { Phenolic acids, Flavonoids, Tannins, Querceti, } \\
\text { Epicatechin, Glycosides, Diverse minerals (Lakkab et } \\
\text { al. 2018) }\end{array}$ & $\begin{array}{l}\text { Anti-inflammatory, Antibacterial, Antioxidant, Anti- } \\
\text { ulcer, Anti-constipation, Anti-diarrheique (Rtibi et } \\
\text { al. 2017) }\end{array}$ \\
\hline & Glycyrrhiza Glabra L. & $\begin{array}{l}\text { Isoniazid, Diethylothluamide, Benzoic acid, Benzene, } \\
\text { Linalol, Prasterone, Warfarin, lodoquinol, phenol } \\
\text { (Chouitah 2012) }\end{array}$ & $\begin{array}{l}\text { Antibacterial (Chouitah 2012) } \\
\text { Antioxidant (Esmaeili et al. 2019) }\end{array}$ \\
\hline & Trigonella foenum-graecum L. & $\begin{array}{l}\text { Proteins, Carbohydrates, Sapogenins, Steroidal } \\
\text { Saponosides, Coumarin, Flavonoids, Amino acids, } \\
\text { Lipids (Ghedira et al. 2010) }\end{array}$ & $\begin{array}{l}\text { Hypocholesterolemic, hypotriglyceridemic } \\
\text { (Ghedira et al. 2010). Antibacterial , Antioxidant, } \\
\text { Hepatoprotective (Meera et al. 2009) }\end{array}$ \\
\hline Juglandaceae & Juglans regia L. & $\begin{array}{l}\alpha \text { - Pinene, } \beta \text { - Pinene, } \beta \text {-caryophyllene, germacrene } D \text {, } \\
\text { limonene (Rather et al. 2012) }\end{array}$ & $\begin{array}{l}\text { Antibacterial, Antioxidant (Rather et al. 2012). } \\
\text { Anticancer (Negi et al. 2011) }\end{array}$ \\
\hline
\end{tabular}




\begin{tabular}{|c|c|c|c|}
\hline Iridaceae & Crocus sativus $\mathrm{L}$. & $\begin{array}{l}\text { Glycosidic carotenoids, Picrocrocin, Trimethyl } \\
\text { hydroxy, Carboxaldehyde cyclohexene, Kkaempherol, } \\
\text { Cistrans-crocins, Safranal. 2-nitroaniline (Caballero- } \\
\text { Ortega et al. 2004) }\end{array}$ & $\begin{array}{l}\text { Anxiolytic, Antidepressant (De Monte et al. 2014) } \\
\text { Antioxidant, Anti-tumor, Anti- inflammatory } \\
\text { (Khorasanchi et al. 2018) }\end{array}$ \\
\hline \multirow[t]{5}{*}{ Lamiaceae } & Lavandula dentata L. & $\begin{array}{l}\text { Polyphenols, Flavonoids, Tannins, Anthracene } \\
\text { derivatives, Reducing compounds, Terpenoids } \\
\text { (Bachiri et al. 2016) }\end{array}$ & $\begin{array}{l}\text { Antibacterial (Bachiri et al. 2016) } \\
\text { Antioxidant, Anti-Inflammatory, Hepatoprotective } \\
\text { (Pereira et al. 2019) }\end{array}$ \\
\hline & Mentha pulegium $\mathrm{L}$. & $\begin{array}{l}\text { Polyphenols, Piperitone, Piperitenone, a-Terpineol, } \\
\text { Pulegone (Mahboubi \& Haghi 2008) }\end{array}$ & $\begin{array}{l}\text { Anti-Hepatic, Antibacterial, Antioxidant, Anti- } \\
\text { myometrium, Relaxant, Hepatotoxicity (Hadi at al. } \\
\text { 2017). }\end{array}$ \\
\hline & Ocimum basilicum L. & $\begin{array}{l}\text { Linalool, 1,8-Cineol, Eugenol, Methyl cinnamate, Iso } \\
\text { caryophyllene, a -cubebene (Ismail. 2006) }\end{array}$ & $\begin{array}{l}\text { Analgesic, Anti-inflammatory, Hypoglycemic, } \\
\text { Antihyperlipidemic, Antiulcerative, } \\
\text { Cardioprotective, Stimulant Activity, Sedative, } \\
\text { Hypnotic, Anticonvulsant, Antibacterial, Anticancer } \\
\text { (Ch et al. 2015) }\end{array}$ \\
\hline & Origanum compactum Benth & $\begin{array}{l}\text { Polyphenols, Flavonoids (Bouyahya et al. 2017) } \\
\text { Sesquiterpenes, Monoterpenes, Carvacrol, } \beta \text { - } \\
\text { Cymene, Thymol, } y \text {-Terpinene (Laghmouchi et al. } \\
\text { 2018) }\end{array}$ & $\begin{array}{l}\text { Antibacterial, Antioxidant, Antileishmanial } \\
\text { (Bouyahya et al. 2017) }\end{array}$ \\
\hline & Salvia officinalis $\mathrm{L}$. & $\begin{array}{l}\text { Camphor, } \alpha \text {-Thujone, 1,8-Cineole, Viridiflorol, } \beta- \\
\text { Thujone, } \beta \text {-caryophyllene (Khedher et al. 2017) }\end{array}$ & $\begin{array}{l}\text { Antioxidant, Antibacterial, Insecticidal, Allelopathic } \\
\text { (Khedher et al. 2017) }\end{array}$ \\
\hline Lauraceae & Cinnamomum verum J. Presl & $\begin{array}{l}\text { Cinnamaldehyde, Eugenol, Caryophyllene, Cinnamyl } \\
\text { acetate, Xinnamic acid, a-Humulene } \\
\text { monoterpenes, Diterpenes, Sesquiterpenes, } \\
\text { Oxygenated hydrocarbons, Polyphenols (Singh et al. } \\
\text { 2020) }\end{array}$ & $\begin{array}{l}\text { Antioxidant, Antibacterial, Anti-inflammatory, } \\
\text { Anticancer, Antidiabetic, Wound healing, Anti-HIV, } \\
\text { Anti-anxiety, Antidepressant (Singh et al. 2020) } \\
\text { Strengthens the immune system (Khanal et al. } \\
\text { 2020) }\end{array}$ \\
\hline Lythraceae & Punica granatum L. & $\begin{array}{l}\text { Phenolics, Favonoids, Anthocyanins, Tannins (El Kar } \\
\text { et al. 2011) }\end{array}$ & $\begin{array}{l}\text { Antimicrobial, Antioxidant (Iskounen \& Tadount } \\
\text { 2018). Anti-inflammatory (Benzaioua et al. 2019) }\end{array}$ \\
\hline Moraceae & Ficus carica L. & $\begin{array}{l}\text { Fatty acids, Flavonoids, Phenolic compounds, } \\
\text { Phytosterols, Anthocyanin (Badgujar et al. 2014) }\end{array}$ & $\begin{array}{l}\text { Antipyretic, Anti-inflammatory, Antispasmodic, } \\
\text { Antihelmintic, Hepatoprotective, Anticonstipation, } \\
\text { Hypoglycemic, Anticancer, Antiviral, } \\
\text { Antimutagenic, Anti-angiogenic, Antibacterial, } \\
\text { Antioxidant (Badgujar et al. 2014) }\end{array}$ \\
\hline Myrtaceae & Eucalyptus globulus Labill & $\begin{array}{l}\text { 1,8-Cineole, } \alpha \text {-Pinene, } \alpha \text {-terpineol acetate, } \beta \text {-Pinene, } \\
\text { Sabinene, Limonene, Isoledene, } \alpha \text {-Gurjunene, } \\
\text { Alloaromadendrene (Abdossi et al. 2015) }\end{array}$ & $\begin{array}{l}\text { Antibacterial, Antioxidant (Dezsi et al. 2015) } \\
\text { Febrifuges, Tonics, Astringents, Antiseptics, } \\
\text { Hemostats, Dewormers (Boukhatem et al. 2018) }\end{array}$ \\
\hline
\end{tabular}




\begin{tabular}{|c|c|c|c|}
\hline & Eugenia caryophyllata Thunb & $\begin{array}{l}\text { Eugenol, Eugenyl Acetate, } \beta \text {-Caryophyllene, 2- } \\
\text { Heptanone, Ethyl Hexanoate, Humulenol, A - } \\
\text { Humulene, Calacorene, Calamenene (Chaieb et al. } \\
\text { 2007) }\end{array}$ & $\begin{array}{l}\text { Antibacterial, Antioxidant, Antiviral, } \\
\text { Antiinflammatory, Cytotoxic, Insect repellent, } \\
\text { Anaesthetic properties (Chaieb et al. 2007) }\end{array}$ \\
\hline Nitrariaceae & Peganum harmala L. & $\begin{array}{l}\text { Harmaline, Harmine, Harmalol, Harman, Quinazoline } \\
\text { derivatives, Vasicine, Vasicinone, Anthroquinons } \\
\text { (Asgarpanah \& Ramezanloo 2012) }\end{array}$ & $\begin{array}{l}\text { Antibacterial, Antifungal, Antitumor, Antidiabetic, } \\
\text { Antioxidant, Antiviral (Asgarpanah \& Ramezanloo } \\
\text { 2012) }\end{array}$ \\
\hline Oleaceae & Olea europaea L. & $\begin{array}{l}\text { Alkaloids, Flavonoids, Tannins, Coumarins, Free } \\
\text { quinones, Sterols, triterpenes, Terpenoids, } \\
\text { Saponosides, Glycosides, Reducing compounds } \\
\text { (Himour et al. 2016) }\end{array}$ & $\begin{array}{l}\text { Antioxidant, Antithrombotic, Antiatherogenic, Anti- } \\
\text { Inflammatory (Nasopoulou et al. 2014) }\end{array}$ \\
\hline Pinaceae & $\begin{array}{l}\text { Cedrus atlantica } \\
\text { Manetti ex Endl. }\end{array}$ & $\begin{array}{l}\text { a-Himachalene, } \beta \text {-Himachalene, } \gamma \text {-Himachalene, } \\
\text { Cedrol, Isocedranol, } \alpha \text {-Pinene (Zoubi et al. 2017) }\end{array}$ & Antibacterial, Insecticide (Fidah et al. 2016) \\
\hline Piperaceae & Piper nigrum & $\begin{array}{l}\text { Ethanol, Ethyl acetate, } \beta \text {-Caryophylline, Limonene, } \beta \text { - } \\
\text { Pinene, Sabinene (Kapoor et al. 2009) }\end{array}$ & $\begin{array}{l}\text { Antihypertensive, Anti-asthmatic, Antibacterial, } \\
\text { Antioxidant, Anti-cancer, Anti-inflammatory, } \\
\text { Hepatoprotective, Anti-diarrheal, Antidepressant, } \\
\text { Anticonvulsant, Analgesic, Immunomodulatory, } \\
\text { Fertility activity (Damanhouri \& Ahmad 2014) } \\
\text { Strengthens the immune system (Khanal et al. } \\
\text { 2020) }\end{array}$ \\
\hline Plantaginaceae & Plantago major $\mathrm{L}$. & $\begin{array}{l}\text { Polysaccharides, Lipids (saturated and non- } \\
\text { saturated), Amino acids (essential and non-essential), } \\
\text { Caffeic acid derivatives, Flavonoids, Iridoidglycosides, } \\
\text { Terpenoids, Phenols (ferulic acid), Tannins } \\
\text { (Nazarizadeh et al. 2013) }\end{array}$ & $\begin{array}{l}\text { Anti-inflammatory, Antibacterial, Antitumor, Anti- } \\
\text { Fatigue, Anti-ulcerogenic, Antidiarrheal, } \\
\text { Antinociceptive, Antioxidant, Hepatoprotective, } \\
\text { Hematopoietic, Immune Enhancing, Antigenotoxic } \\
\text { Effects (Nazarizadeh et al. 2013) }\end{array}$ \\
\hline Poaceae & Hordeum vulgare $\mathrm{L}$. & Phenols, Flavonoids, Tannins (Yousra 2017) & Antioxidant, Antibacterial (Yousra 2017) \\
\hline Portulacaceae & Portulaca oleraceae L. & $\begin{array}{l}\text { Flavonoids, Alkaloids, Fatty acids, Terpenoids, } \\
\text { Polysaccharides, Vitamins, Sterols, Proteins, } \\
\text { Minerals, Amino acids (Zhou et al. 2015) }\end{array}$ & $\begin{array}{l}\text { Antibacterial, Antiulcerogenic, Anti-inflammatory, } \\
\text { Antioxidant, Anticancer, Neuroprotective activity } \\
\text { (Zhou et al. 2015) }\end{array}$ \\
\hline Ranunculaceae & Nigella damascena L. & $\begin{array}{l}\text { Phenols, Flavonoids, Volatile oils, Alkaloids, fatty } \\
\text { acids, Lipids, Sterols, vitamins and } \beta \text {-carotene (Sobhi } \\
\text { 2018) }\end{array}$ & $\begin{array}{l}\text { Antitumor, Antioxidant, Antibacterial, Anti- } \\
\text { Inflammatory, Analgesic, Antiparasitic, Antiulcer, } \\
\text { Antidiabetic, Stimulating effect of the immune } \\
\text { system (Ghedira \& Jeune 2010) } \\
\text { Anti-allergic And Anti-histamine, Hepato- } \\
\text { protective, Antitoxic (Sobhi 2018) }\end{array}$ \\
\hline
\end{tabular}




\begin{tabular}{|c|c|c|c|}
\hline Salicaceae & Populus alba L. & $\begin{array}{l}\text { 1,8-Cineole, Eudesmol, } \delta \text {-Cadinene, sesquiterpenes, } \\
\alpha \text {-Eudesmol, } \beta \text {-Eudesmol, Linalool, } \beta \text {-Cyclocitral, } \\
\text { Methyl Eugenol, Salicylic aldehyde (Belkhodja et al. } \\
2016 \text { ) }\end{array}$ & $\begin{array}{l}\text { Cytotoxic, Antioxidant, Antiproliferative (Gezici et } \\
\text { al. 2017). Anti-inflammatory, Antibacterial, Antiviral } \\
\text { (Silici \& Kutluca 2005) }\end{array}$ \\
\hline Verbenaceae & Aloysia triphylla (L'Herit.) Britton & $\begin{array}{l}\text { Limonene, } \beta \text {-Caryophyllen, p-Cymene, Camphor, } \\
\text { Linalool, a-Pinene, Thymol, Hernandulcin, } \\
\text { Epihernandulcin, Phenolic acids (Pascual et al. 2001) }\end{array}$ & $\begin{array}{l}\text { Antibacterial, Hypotensive, Anti-inflammatory } \\
\text { (Pascual et al. 2001) }\end{array}$ \\
\hline Vitaceae & Vitis vinifera $\mathrm{L}$. & $\begin{array}{l}\text { Carotenoids, Tocopherols, Glycosylated, Hyperoside } \\
\text { flavonoids, Rutin, Ellagic, Chlorogenic acids (Aubert \& } \\
\text { Chalot 2018) }\end{array}$ & $\begin{array}{l}\text { Antioxidant, Antibacterial, Neuroprotective, } \\
\text { Cytotoxic assays (Aubert \& Chalot 2018) } \\
\text { Strengthens the immune system (Khanal et al. } \\
\text { 2020) }\end{array}$ \\
\hline \multirow[t]{2}{*}{ Zingiberaceae } & Curcuma longa L. & $\begin{array}{l}\text { Demethoxycurcumin, Bisdemethoxycurcumin, } \\
\text { Curcumins, Turmerin, Amino acid, Carotenoids } \\
\text { (Niranjan \& Prakash 2008) }\end{array}$ & $\begin{array}{l}\text { Anti-inflammatory, Antibacterial, } \\
\text { Anti-parasitic, Antispasmodic, Antioxidant, Anti- } \\
\text { HIV, Anti-tumor, Nematocidal, Gastrointestinal } \\
\text { activities (Araujo \& Leon 2001) } \\
\text { Strengthens the immune system (Khanal et al. } \\
\text { 2020) }\end{array}$ \\
\hline & Zingiber officinale Roscoe & $\begin{array}{l}\text { Camphene, p-cineole, R-Terpineol, Zingiberene, } \\
\text { Pentadecanoic acid, Cuminal, y-Terpinene, } \\
\text { Pinocarveol (Bellik 2014) }\end{array}$ & $\begin{array}{l}\text { Antioxidant, Antibacterial, Anticancer (Oueslati et } \\
\text { al. 2018) } \\
\text { Strengthens the immune system (Khanal et al. } \\
\text { 2020) }\end{array}$ \\
\hline
\end{tabular}




\section{Medicinal plants for therapeutic uses}

This study identified 46 species belonging to 30 families and 43 genera at the end of this survey. Eleven families were represented by $54.34 \%$ of the total number of species used for protection against Covid-19: Lamiaceae (5 species), Asteraceae (3), Fabaceae (3), Amaryllidaceae (2), Apiaceae (2), Araliaceae (2), Brassicaceae (2), Cupressaceae (2), Myrtaceae, and Zingiberaceae (2). The other families are represented by one plant species. Algeria by Helali et al. (2020) showed that Lamiaceae was most importnat in order to prevent infection with Covid-19.

The data also showed that 25 medicinal plants promote immunity, 18 species to relieve some symptoms related to respiratory infection caused by SARS-CoV-2, and 11 species to cleanse the area. We gave each listed plant the scientific name, the family, the common name, the part used, the preparation method, and the values: F, VI, FRC, P1, P0, and RP (Table 2). Also, the chemical and biological data of these plants are shown in Table 3 . The most commonly used parts of these plants are their leaves, followed by the fruits and seeds. One organ predominance use over another in the therapeutic field derives from its concentration in active ingredients. The leaves are the most widely used because they are at the same time a seat of photochemical reactions and a reservoir of organic matter derived from them (Babba Aissa et al. 1999). The recommended recipes are mostly prepared by decoction. Most of these preparations are prescribed in precise doses $(61 \%)$. These results are in agreement with the study carried out by El Alami et al. 2020, who found that infusion and decoction were the most modes of preparation of medicinal plants usable for prevention during the Covid-19 pandemic in Morocco.

The majority of users considered that high doses can be toxic and even fatal. These results can be explained by the fact that most of the respondents have medium and university education, which is reflected in their use of plants under strict use and precise doses. The majority of informants (48\%) acquired their knowledge about medicinal plants through hereditary transmission and ancestors' support. In comparison, 38\% based their knowledge on own experience and the media, while $14 \%$ took their knowledge from herbalists.

\section{Medicinal plants for widespread use}

The lack of specific treatment to protect the population from this devastating virus has prompted many Moroccan to seek solutions for interventions that can help to prevent or better manage the epidemic. Medicinal plants contain a wide range of chemicals that can exert different biological effects.
The active ingredients are classified into several groups according to their chemical composition. Among the main groups are: Polyphenols are having a multitude of biological activities in relation to their chemical structure. These molecules show anticarcinogenic, anti-inflammatory, antiatherogenic, anti-thrombotic, analgesic, antibacterial, antiviral, anticancer, anti-allergenic, and vasodilator activities (Falleh et al. 2008). Alkaloids, these molecules act at the level of the autonomic nervous system: sympathomimetic (ephedrine) or sympatholytic, anticholinergic, and ganglioplegic (Bruneton 1999). Flavonoids are products that are widely distributed in the plant kingdom and can exert several biological properties, including antioxidant, anti-hepatotoxic, anti-allergic, antibacterial, antiviral, antiinflammatory, and cytotoxic activity (Ghedira 2005).

Based on the data collected, it was found that 25 medicinal plants are used locally to strengthen the immunity system, allowing the body to resist this virus's attack better. The most cited species are Zingiber officinale Roscoe (RFC=0.79), Olea europaea L. (RFC=0.76), Vitis vinifera $\mathrm{L}$. (RFC=0.74), and Allium sativum L. (RFC=0.74). Pukar et al. (2020), identifies the formulation of individual medicinal plants capable of modulating voices related to immune system enhancement and progression of the pathogenesis of multiple diseases against Covid-19. Among these plants, four species are cited by our informants (Cinnamomum verum, Piper nigrum, Vitis vinifera, Turmeric long, and Zingiber officinale). The decoction of the blend of Ocimum tenuiflorum, Cinnamomum verum, Piper nigrum, Zingiber officinale, and Vitis vinifera has been prescribed by the Service of AYUSH as a preventive measure to enhance immunity and inhibit the severity of infection caused by Coronavirus (Pukar et al.2020). Allium sativum was prescribed to strengthen immunity. In vitro study showed that garlic extricate represses inhibits infection by restraining viral nucleoprotein union and polymerase action (Babich et al. 2020). Ghedira (2005) carried out another study that determined the importance of Flavonoids as immunostimulants, especially in immunocompromised subjects. The analysis of Table 3 showed the presence of this molecule in several species (Carum carvi, Hedera helix, Artemisia absinthium, Arbutus unedo, Ceratonia siliqua, and Trigonella foenum-graecum ...). These species are rich in chemicals in addition to vitamins, minerals, and proteins. These contents may justify their use to stimulate defense systems against microbial and viral attacks by the surveyed population. Thus 18 species are used to relieve some respiratory infection symptoms, and the most cited are Eucalyptus globulus, Trigonella foenumgraecum, and Aloysia triphylla. These plants are known for their richness in essential oils; hence 
they're antibacterial and antioxidant activities demonstrated by numerous in vitro and in vivo studies. On the other hand, the plants utilized in traditional medicine against Covid-19 in subSaharan Africa, such as Allium cepa are also often used to treat influenza-related fever (Vroh 2020). Moreover Boskabady et al. (2020) showed that the roots of Curcumin have a diminishing and preventive impact on respiratory disorders. The other 11 species of plants are used to sanitize the area. The most frequently used are Eucalyptus globulus, Lavandula denta, and Eugenia caryophyllata. A study conducted in Algeria on plants used to prevent infection by COVID-19, showed that 24 plants are used to clean the air, of which Eucalyptus globulus is the most cited. It should be noted also that among the 57 plants cited by the respondents to our survey, six species have proven antiviral activity (Table 3 ).

For the species with the highest value of importance (VI), the attention is focused on the Zingiber officinale. This kind is very rich in vitamins that are essential for the functioning of the immune system. Eucalyptus globulus, a species-rich in eucalyptol (80-85), are widely used against respiratory problems, incredibly to cleanse and thin the lung mucous membranes. Eucalyptus is also used for rubbing, inhalation, fumigation, and diffusion in the atmosphere (Tachema et al. 2020).

Most of these plants used by the Moroccan population during this pandemic are applied to fight many respiratory tract infections that cause manifestations similar to those of the coronavirus. These species are known for their richness in essential oils, flavonoids, alkaloids, glycosides, aromatic constituents, phenolic lipids, carotenoids, many other anti-infectious compounds, and antibacterial activities have been demonstrated by numerous in vitro and in vivo studies (Table 3). The estimated prevalence showed that the proportion of the number of Covid-19 cases observed in the population survey was $11.21 \%$. The prevalence ratio: $\mathrm{RP}=0.126$, which means that an exposed subject's risk is 0.126 times that of an unexposed subject, or an exposed subject has a $35 \%$ lower risk of being ill with COVID 19 than an unexposed subject (Feingold 1998). In the race against time to find a cure for Covid-19, the main advantage would be the fastest treatment for patients. According to most participants, early intake of these plants would avoid complications leading to hospitalization or even a transfer to intensive care. Besides, many of these species have already proven their effectiveness against respiratory and lung diseases. Moreover, four species are effective in strengthening the immune system (Table 3).

\section{Conclusion}

Phytotherapy has been practiced in all regions of Morocco since ancient times. With the new SARSCOV2 pandemic causing acute infectious pneumonia, and in the absence of a specific antiviral for its treatment, medicinal plants are the common option of patients for supportive care to improve the symptoms related to this virus. The current survey results showed that $67.04 \%$ of participants has reported that they are received herbal treatment at the onset of the outbreak, whether to promote immunity, clean the air, or relieve some of the symptoms associated with the respiratory infection that is caused by Coronavirus. The results of our investigation and the gathered information on the most abundant secondary metabolites confirm that they contain several bioactive substances that are known in modern medicine for their biological activities. Plants' complex secondary metabolism has been actually, the source of countless medicinal compounds and has led to drug discovery. Therefore, it is not surprising that plant products and their analogs have been used as the first line of defense against COVID19.

\section{Declarations}

List of abbreviations: FC: Citation frequency, RFC: Relative citation frequency, Vue: Use value by species, VI: importance value, P: Prevalence estimate, RP: Prevalence ratio, SPSS: Social Science Statistical Software.

Ethics approval and consent to participate: The study was approved by the Department of Biology of the University Ibn Tofail Kenitra. All respondents gave their prior informed consent.

Consent for publication: Not applicable

Competing interests: The authors declare no competing interest.

Authors' contributions: SB: Sent the questionnaire sheets to the respondents, compiled the documentary sources, data analysis, and interpretation, and wrote the manuscript. LZ: Sent the questionnaire sheets to the respondents, designed the plant species identification, and contributed substantially to the data analysis, production, and evaluation of the manuscript. All authors participated in the writing of the manuscript, provided comments on the manuscript, and approved the final version of the manuscript.

\section{Acknowledgments}

We would like to thank everyone who has agreed to answer our questionnaire.

\section{Literature cited}

Abdossi V, Moghaddam EY, Hadipanah A. 2015. Chemical composition of Eucalyptus globulus grown in Iran: In Biological Forum 7(2):322-324. 
Akrout A, El Jani H, Amouri S, Neffati M. 2010. Screening of antiradical and antibacterial activities of essential oils of Artemisia campestris L., Artemisia herba alba Asso, \& Thymus capitatus Hoff. et Link. growing wild in the Southern of Tunisia. Recent Research in Science and Technology 2(1):29-39.

Ali EM. 2013. Phytochemical composition, antifungal, antiaflatoxigenic, antioxidant, and anticancer activities of Glycyrrhiza glabra L. and Matricaria chamomilla L. essential oils. Journal of Medicinal Plants Research 7(29):2197-2207.

Ambe AS, Ouattara D, Tiebre MS, Vroh BTA, Zirihi GN, N'guessan KE. 2015. Diversité des plantes médicinales utilisées dans le traitement traditionnel de la diarrhée sur les marchés d'Abidjan (Côte d'Ivoire). Journal of Animal and Plant Sciences 26(2):4081-4096.

Anita P, Sivasamy S, Kumar PM, Balan IN, Ethiraj S. 2014. In vitro antibacterial activity of Camellia sinensis extract against cariogenic microorganisms: Journal of basic and clinical pharmacy 6(1):35.

Araujo CAC, Leon LL. 2001. Biological activities of Curcuma longa L. Memórias do Instituto Oswaldo Cruz 96(5):723-728.

Asgarpanah J, Ramezanloo F. 2012. Chemistry, pharmacology and medicinal properties of Peganum harmala L. African Journal of Pharmacy and Pharmacology 6(22):1573-1580.

Aubert C, Chalot G. 2018. Chemical composition, bioactive compounds, and volatiles of six table grape varieties (Vitis vinifera L.): Food chemistry 240:524533.

Babba Aissa F. 1999. Flore d'Algérie et du Maghreb. Encyclopédie des plantes utiles. Substances végétales d'Afrique, d'Orient et d'Occident. Ed. Librairie Moderne Rouiba, EDAS, Alger, 368 p.

Babich O, Sukhikh S, Prosekov A, Asyakina L, Ivanova S. 2020. Medicinal plants to strengthen immunity during a pandemic. Pharmaceuticals 13(10) :313.

Bachiri L, Echchegadda G, Ibijbijen J, Nassiri L. 2016. Etude Phytochimique et Activité Antibactérienne de deux espèces de Lavande autochtones Au Maroc : Lavandula stoechas L. et Lavandula dentata L. European Scientific Journal 12(30):313-333.

Badgujar SB, Patel VV, Bandivdekar AH, Mahajan RT. 2014. Traditional uses, phytochemistry and pharmacology of Ficus carica: A review. Pharmaceutical Biology 52(11):1487-1503.

Barrero AF, Herrador MM, Arteaga JF, Akssira M, Mellouki F, Belgarrabe A, Blázquez MA. 2005. Chemical composition of the essential oils of Pistacia atlantica Desf. Journal of Essential Oil Research 17(1):52-54.

Belhaj S, Dahmani J, Belahbib N, Zidane L. 2020. Ethnopharmacological and Ethnobotanical study of Medicinal plants in the Central High Atlas, Morocco. Ethnobotany Research and Applications 20:1-40.

Belkhiri F. 2018. Activité antimicrobienne et antioxydante de deux plantes médicinales: Salvia verbenacea et Lepidium sativum. Doctoral dissertation.

Belkhodja H, Meddah B, Touil AT, Şekeroğlu N, Sonnet P. 2016. Chemical composition and properties of essential oil of Rosmarinus officinalis and Populus alba. World Journal of Pharmacology $5041: 108-119$.

Bellik Y. 2014. Total antioxidant activity and antimicrobial potency of the essential oil and oleoresin of Zingiber officinale Roscoe. Asian Pacific Journal of Tropical Disease 4(1):40-44.

Benhammou N, Bekkara FA, Panovska TK. 2008. Antioxidant and antimicrobial activities of the Pistacia lentiscus and Pistacia atlantica extracts. African Journal of Pharmacy and Pharmacology 2(2):022028.

Benlamdini N, Elhafian M, Rochdi A, Zidane L. 2014. Étude floristique et ethnobotanique de la flore médicinale du Haut Atlas oriental (Haute Moulouya). Journal of Applied Biosciences 78:6771-6787.

Benzaioua R, Boulali Z, Younes C, Hireche SE. 2019. Etude in vitro de l'activité anti-inflammatoire de l'extrait de l'écorce de la plante Punica granatum L. Doctoral dissertation, Université de Jijel.

Blama A, Mamine F. 2013. Etude ethnobotanique des plantes médicinales et aromatiques dans le sud algérien : le Touat et le Tidikelt. In 5 . Symposium international des plantes aromatiques et médicinales, $17 p$.

Boskabady MH, Shakeri F, Naghdi F. 2020. The effects of Curcuma longa L. and its constituents in respiratory disorders and molecular mechanisms of their action. In Studies in Natural Products Chemistry 65:239-269.

Boukhatem MN, Ferhat MA, Kameli A, Mekarnia M. 2018. Eucalyptus globulus (Labill.) : un arbre à essence aux mille vertus. Phytothérapie 16 :203214.

Bouyahya A, Abrini J, Bakri Y, Dakka N. 2017. Screening phytochimique et évaluation de l'activité antioxydante et antibactérienne des extraits d'Origanum compactum. Phytothérapie 15(6):379383. 
Briguiche H, Zidane L. 2019. Etude floristique et ethnobotanique des plantes médicinales utilisées dans le traitement des maladies de l'appareil respiratoire dans la région de Doukkala. Bulletin de I'Institut Scientifique, Rabat, Section Science de la Vie 41:33-41.

Bruneton J. 1999. Pharmacognosie, Phytochimie, Plantes médicinales, $3 e$ edition, Edition Lavoisier TEC et DOC.

Caballero-Ortega $\mathrm{H}$, Pereda-Miranda R, RiverónNegrete L, Hernández JM, Medécigo-Ríos $M$, Castillo-Villanueva A, Abdullaev FI. 2004. Chemical composition of saffron (Crocus sativus L.) from four countries. Acta Horticulturae, 321-326 pp.

Ch MA, Naz SB, Sharif A, Akram M, Saeed MA. 2015. Biological and pharmacological properties of the sweet basil (Ocimum basilicum). Journal of Pharmaceutical Research International, 330-339 pp.

Chaachouay N, Benkhnigue O, Fadli M, El Ibaoui H, El Ayadi R, Zidane L. 2019. Ethnobotanical and Ethnopharmacological Study of Medicinal and Aromatic Plants Used in the Treatment of Respiratory System Disorders in the Moroccan Rif. Ethnobotany Research and Applications 18:1-16.

Chaieb K, Hajlaoui H, Zmantar T, Kahla-Nakbi AB, Rouabhia M, Mahdouani K, Bakhrouf A. 2007. The chemical composition and biological activity of clove essential oil, Eugenia caryophyllata (Syzigium aromaticum L. Myrtaceae): a short review. Phytotherapy Research: An International Journal Devoted to Pharmacological and Toxicological Evaluation of Natural Product Derivatives 21(6):501506.

Chouitah O. 2012. Composition chimique et activité antibactérienne des huiles essentielles des feuilles de Glycyrrhiza glabra. Doctoral dissertation, Université d'Oran.

Damanhouri ZA, Ahmad A. 2014. A review on therapeutic potential of Piper nigrum L. Black Pepper: The King of Spices Med Aromat Plants: 3161.

De Monte C, Carradori S, Chimenti P, Secci D, Mannina L, Alcaro F, Alcaro S. 2014. New insights into the biological properties of Crocus sativus L.: chemical modifications, human monoamine oxidases inhibition and molecular modeling studies: European journal of medicinal chemistry 82:164-171.

Dezsi Ș, Bădărău AS, Bischin C, Vodnar DC, SilaghiDumitrescu R, Gheldiu AM, Vlase L. 2015. Antimicrobial and antioxidant activities and phenolic profile of Eucalyptus globulus Labill, and Corymbia ficifolia (F. Muell.) KD Hill \& LAS Johnson leaves. Molecules 20(3):4720-4734.
Dossou ME, Houessou GL, Lougbégnon OT, Tenté AHB, Codjia JTC. 2012. Etude ethnobotanique des ressources forestières ligneuses de la forêt marécageuse d'Agonvè et terroirs connexes au Bénin. Tropicultura: 30-31.

Drider NEH, Kada F. 2019. Etude des activités antibactériennes et antifongiques des huiles essentielle de Salvia officinalis, Juniperus phoenicea etMentha puleiuim. Bouira, Algerie.

Eddouks M, Lemhadri A, Michel JB. 2004. Caraway and caper: potential anti-hyperglycaemic plants in diabetic rats. Journal of Ethnopharmacology 94(1):143-148.

El Alami A, Fattah A, Chait A. 2020. Medicinal plants used for the prevention purposes during the covid-19 pandemic in Morocco. Journal of Analytical Sciences and Applied Biotechnology 2(1) :2-1.

El Kar C, Ferchichi A, Attia F, Bouajila J. 2011. Pomegranate (Punica granatum) juices: chemical composition, micronutrient cations, and antioxidant capacity. Journal of food Science 6(6):795-800.

El Kolli M. 2018. Composition chimiques et activités biologiques des huiles essentielles de quelques apiacees Carum montanum Benth. \& Hook et Daucus gracilus stem. Université Sétif, Algérie.

El-Hadri Y. 2019. Contribution à l'étude ethnobotanique des plantes médicinales utilisées dans la région de Beni Mellal-Khénifra. Bouira, Algerie.

El-Sawi SA, Motawae HM, Ali AM. 2007. Chemical composition, cytotoxic activity and antimicrobial activity of essential oils of leaves and berries of Juniperus phoenicea L. grown in Egypt. African Journal of Traditional, Complementary and Alternative Medicines 4(4):417-426.

Esmaeili H, Karami A, Hadian J, Saharkhiz MJ, Ebrahimi SN. 2019. Variation in the phytochemical contents and antioxidant activity of Glycyrrhiza glabra populations collected in Iran. Industrial Crops and Products 137:248-259.

Fadili K, Zerkani H, Amalich S, Zair T. 2017. Etude phytochimique et evaluation de l'activite antioxydante des feuilles et des fruits du Capparis spinosa I. phytochemical study and evaluation of antioxidant activity of leaves and fruits of Capparis spinosa L. American Journal of Innovative Research and Applied Sciences 5(2):108-118.

Falleh H, Ksouri R, Chaieb K, Karray-Bouraoui N, Trabelsi N, Boulaaba M, Abdelly C. 2008. Phenolic composition of Cynara cardunculus L. organs and their biological activities: Comptes Rendus Biologies 331(5):372-379. 
Feingold J. 1998. A propos de l'estimation de la prévalence et de l'incidence des maladies héréditaires. Médecine et Sciences 14: 1402-1405

Fidah A, Salhi N, Rahouti M, Kabouchi B, Ziani M, Aberchane M, Famiri A. 2016. Natural durability of Cedrus atlantica wood related to the bioactivity of its essential oil against wood decaying fungi. Maderas Ciencia y Tecnología 18(4):567-576.

Fouad Z, Lahcen Z. 2020. Antidiabetic medicinal plants in Morocco: ethnobotanical survey of the population of béni mellal. Plant Archives 20(1):337343.

Gezici S, Sekeroglu N, Kijjoa A. 2017. In vitro anticancer activity and antioxidant properties of essential oils from Populus alba L. and Rosmarinus officinalis L. from South Eastern Anatolia of Turkey. Indian Journal of Pharmaceutical Education and Research 51(3):498-503.

Ghedira K, Goetz P. 2013. Cucurbita pepo L. (Cucurbitaceae) Graine de courge ou citrouille. Phytothérapie 11(1):46-51.

Ghedira K, Goetz PLJR, Le Jeune R. 2010. Fenugrec : Trigonella fœnum-græcum L. (Fabaceae ex. Leguminosae). Phytothérapie 8(3):180-184.

Ghedira K, Le Jeune R. 2010. Huile de nigelle cultivée, Nigella sativa L. (Ranunculaceae). Phytothérapie 8(2):124-128.

Ghedira K. 2005. Les flavonoïdes : structure, propriétés biologiques, rôle prophylactique et emplois en thérapeutique. Phytothérapie 3(4) :162169.

Goetz P. 2004. Mode d'emploi du ginseng et d'autres adaptogènes. Phytothérapie, 2(4):113-116.

Hadi MY, Hameed IH, Ibraheam IA. 2017. Mentha pulegium: Medicinal uses, Anti-Hepatic, Antibacterial, Antioxidant effect and Analysis of Bioactive Natural Compounds: A Review. Research Journal of Pharmacy and Technology 10(10):35803584.

Helali A, Mokhtari C, Ghoul M, Belhadef MS. 2020. Prévenir l'infection par le COVID19 : quelle place pour les plantes médicinales selon la population Algérienne ? Algerian Journal of Pharmacy 3(1):2602-795X.

Himour S, Yahia A, Belattar H, Bellebcir L. 2016. Etude phytochimique des feuilles d'Olea europaea $\mathrm{L}$. var Chemlel d'Algérie. J. of Biores. Valorization 1(1):34-38.

Houmenou V, Adjatin A, Tossou MG, Yedomonhan H, Dansi A, Gbenou J, Akoegninou A. 2017. Etude ethnobotanique des plantes utilisées dans le traitement de la stérilité féminine dans les départements de l'Ouémé et du plateau au Sud Bénin. International Journal of Biological and Chemical Sciences 11(4):1851-1871.

Huang C, Wang Y, Li X, Ren L, Zhao J, Hu Y, Cheng Z. 2020. Clinical features of patients infected with 2019 novel coronavirus in Wuhan, China. The Lancet 395(10223):497-506.

Idm'hand E, Msanda F, Cherifi K. 2019. Étude ethnobotanique des plantes médicinales utilisées dans le traitement de la lithiase urinaire dans la province de Tarfaya (Maroc). International Journal of Innovation and Applied Studies 26(3):711-719.

Iskounen T, Tadount S. 2018. L'activité antioxydante et antimicrobienne de l'extrait de l'écorce et du jus de la grenade Punica granatum de Kabylie, variété Lahlou. I Université Mouloud Mammeri, Algérie.

Ismail M. 2006. Central properties and chemical composition of Ocimum basilicum essential oil: Pharmaceutical Biology 44(8):619-626.

Kapoor IPS, Singh B, Singh G, De Heluani CS, De Lampasona MP, Catalan CA. 2009. Chemistry and in vitro antioxidant activity of volatile oil and oleoresins of black pepper (Piper nigrum): Journal of Agricultural and Food Chemistry 57(12):5358-5364.

Khanal P, Duyu T, Dey YN, Patil BM, Pasha I, Wanjari M. 2020. Network pharmacology of AYUSH recommended immune-boosting medicinal plants against COVID-19. Journal of Ayurveda and Integrative Medicine, doi: 10.1016/j.jaim.2020.11.004.

Khedher MRB, Khedher SB, Chaieb I, Tounsi S, Hammami M. 2017. Chemical composition and biological activities of Salvia officinalis essential oil from Tunisia: Excli Journal 16: 160.

Khorasanchi Z, Shafiee M, Kermanshahi F, Khazaei M, Ryzhikov M, Parizadeh MR, Hassanian SM. 2018. Crocus sativus a natural food coloring and flavoring has potent anti-tumor properties. Phytomedicine 43 :21-27.

Krati K. 1991. L'utilisation des plantes médicinales pour le traitement des troubles fonctionnels intestinaux dans le contexte marocain. Marrakech, Maroc.

Laghmouchi Y, Belmehdi O, Senhaji NS, Abrini J. 2018. Chemical composition and antibacterial activity of Origanum compactum Benth, essential oils from different areas at northern Morocco: South African Journal of Botany 115:120-125.

Lam SK, Ng TB. 2009. A protein with antiproliferative, antifungal and HIV-1 reverse transcriptase inhibitory activities from caper 
(Capparis spinosa) seeds. Phytomedicine 16(5):444-450.

Lim DS, Bae KG, Jung IS, Kim CH, Yun YS, Song JY. 2002. Anti-septicaemic effect of polysaccharide from Panax ginseng by macrophage activation. Journal of Infection 45(1):32-38.

Mahboubi M, Haghi G. 2008. Antimicrobial activity and chemical composition of Mentha pulegium $\mathrm{L}$. essential oil: Journal of Ethnopharmacology 119(2):325-327.

Mahboubi M, Mahboubi M. 2015. Chemical composition, antimicrobial and antioxidant activities of Eugenia caryophyllata essential oil: Journal of Essential Oil-Bearing Plants 18(4):967-975.

Mansouri N, Satrani B, Ghanmi M, El Ghadraoui L, Guedira A, Aafi A. 2011. Composition chimique, activité antimicrobienne et antioxydante de l'huile essentielle de Juniperus communis du Maroc. Bulletin de la société royale des sciences de liège. Bulletin de la Société Royale des Sciences de Liège. Centre de Recherche Forestière, Maroc, 791-805.

Marongiu B, Porcedda S, Piras A, Sanna G, Murreddu M, Loddo R. 2006. Extraction of Juniperus communis L. ssp. nana Willd, essential oil by supercritical carbon dioxide: Flavour and Fragrance Journal 21(1):148-154.

Meera R, Devi P, Kameswari B, Madhumitha B, Merlin NJ. 2009. Antioxidant and hepatoprotective activities of Ocimum basilicum Linn and Trigonella foenum-graecum Linn against $\mathrm{H} 2 \mathrm{O} 2$ and $\mathrm{CCl} 4$ induced hepatotoxicity in goat liver. Indian Journal of Experimental Biology 47:584-590.

Ministère de la Santé. 2020. Le Portail Officiel du Coronavirus au Maroc. Maladie due au nouveau Coronavirus : la veille sanitaire au Maroc. http://www.covidmaroc.ma/pages/Accueil.aspx.

Mnayer D, Fabiano-Tixier AS, Petitcolas E, Hamieh T, Nehme N, Ferrant C, Chemat F. 2014. Chemical composition, antibacterial and antioxidant activities of six essentials oils from the Alliaceae family. Molecules 19(12):20034-20053.

Moumene F, Benali-Toumi F, Benabderrahman M, Benyamina A, Selem H, Dif MM. 2016. Composition chimique et activité antibactérienne des huiles essentielles d'Allium vineale et Allium sativum de l'Ouest Algérien. Phytothérapie 14(3):170-175.

Moumni M, Elwatik L, Kasimi AR, Homrani Bakali AM. 2013. Induction du chemotype a davone de I'huile Essentielle d'armoise blanche (Artemisia herba alba) par domestication à Errachidia (Sud-Est du Maroc). Science Lib, 2013a, 5.

Nasopoulou C, Karantonis HC, Detopoulou M, Demopoulos CA, Zabetakis I. 2014. Exploiting the anti-inflammatory properties of olive (Olea europaea) in the sustainable production of functional food and nutraceuticals: Phytochemistry Reviews 13(2):445458.

Nazarizadeh A, Mikaili P, Moloudizargari M, Aghajanshakeri S, Javaherypour S. 2013. Therapeutic uses and pharmacological properties of Plantago major L. and its active constituents: Journal of Basic and Applied Scientific Research 3(9):212221.

Ndjouondo GP, Ngene JP, Ngoule CC, Kidik P, Ndjib $\mathrm{R}$, Dibong SD, Mpondo ME. 2015. Inventaire et caractérisation des plantes médicinales des sous bassins versants Kambo et Longmayagui (Douala, Cameroun). Journal of Animal and Plant Sciences 25(3):3898-3916.

Negi AS, Luqman S, Srivastava S, Krishna V, Gupta N, Darokar MP. 2011. Antiproliferative and antioxidant activities of Juglans regia fruit extracts. Pharmaceutical biology 49(6):669-673.

Nezla S. 2018. Optimisation de l'extraction et étude des activités antibactérienne et anti-hémolytique des protéines de l'ail: Allium sativum. Université de Bouira, Algérie.

Niranjan A, Prakash D. 2008. Chemical constituents and biological activities of turmeric (Curcuma longa I.) a review: Journal of Food Science and Technology 45(2):109.

Oueslati S, Gharsalli W, Abdelkarim M, AissaFennira FBEN, Ksouri R. 2018. Biochemical evaluation and exploration of the antioxidant, antibacterial and anticancer potential of Zingiber officinale Journal of New Sciences 54:3561-3568.

Pascual ME, Slowing K, Carretero E, Mata DS, Villar A. 2001. Lippia: traditional uses, chemistry and pharmacology: a review. Journal of Ethnopharmacology 76(3):201-214.

Pereira OR, Macias RI, Domingues MR, Marin JJ, Cardoso SM. 2019. Hepatoprotection of Mentha aquatica L., Lavandula dentata L. and Leonurus cardiaca L.: Antioxidants 8(8):267.

Qnais E, Raad D, Bseiso Y. 2014. Analgesic and anti-inflammatory effects of an extract and flavonoids from Artemisia herba-alba and their mechanisms of action: Neurophysiology 46(3):238-246.

Rather MA, Dar BA, Dar MY, Wani BA, Shah WA, Bhat BA, Qurishi MA. 2012. Chemical composition, antioxidant and antibacterial activities of the leaf essential oil of Juglans regia L. and its constituents. Phytomedicine 19(13):1185-1190.

Rehman NU, Mehmood MH, Alkharfy KM, Gilani AH. 2012. Studies on antidiarrheal and antispasmodic activities of Lepidium sativum crude extract in rats. 
Phytotherapy Research 26(1):136-141.

Rejdali M. 1995. La flore du Maroc : Etat actuel et perspectives de conservation. Rencontre francophone de Coopération et de partenariat par l'Agence de Coopération Culturelle et Technique et le Maroc, ISBN: 9981-801-26-7; 18-22 pp.

Rhattas M, Douira A, Zidane L. 2016. Étude ethnobotanique des plantes médicinales dans le Parc National de Talassemtane (Rif occidental du Maroc). Journal of Applied Biosciences 97:91879211.

Sasaki JI, Lu C, Machiya E, Tanahashi M, Hamada K. 2007. Processed black garlic (Allium sativum) extracts enhance anti-tumor potency against mouse tumors: Energy (kcal/100 g). Medicinal and Aromatic Journal ofPplant Science and Biotechnology 1(2): 278-281.

Silici S, Kutluca S. 2005. Chemical composition and antibacterial activity of propolis collected by three different races of honeybees in the same region: Journal of Ethnopharmacology 99(1): 69-73.

Singh N, Rao AS, Nandal A, Kumar S, Yadav SS, Ganaie SA, Narasimhan B. 2020. Phytochemical and pharmacological review of Cinnamomum verum J. Presl-a versatile spice used in food and nutrition: Food Chemistry 338:127773.

Sobhi W. 2018. Caractérisation de l'huile des graines de Nigella sativa et étude de son activité hypoglycémiante et son hépato-toxicité. Université de Sétif, Algérie.

Tachema A, Haouatti F, Smail A, Toumi P, Zitouni H. 2020. Plantes et COVID 19 Le receuil des données. EHU.

Tavakol HS, Farzad K, Fariba M, Abdolkarim C, Hassan G, Seyed-Mostafa H Z, Akram R. 2015. Hepatoprotective effect of Matricaria chamomilla. $L$ in paraquat induced rat liver injury. Drug research 65(02):61-64.

Thippeswamy NB, Naidu KA, Achur RN. 2013. Antioxidant and antibacterial properties of phenolic extract from Carum carvi L. Journal of Pharmacy Research 7(4):352-357.

World Health Organization (WHO). 2019. Statement on the second meeting of the International Health: Regulations 2005 Emergency Committee regarding the outbreak of novel coronavirus (2019-nCoV). https://www.who.int/ihr/publications/978924158049 6/en/.

Yousra GR. 2017. Etude comparative de la variabilité interspécifique : morph-phénologique et évaluation de l'activité antioxydante et l'activité biologique chez Triticum durum, Triticum aestivum et Hordeum vulgare. Université des Frères Mentouri
Constantine, Algérie.

Zhou YX, Xin HL, Rahman K, Wang SJ, Peng C, Zhang H. 2015. Portulaca oleracea L.: a review of phytochemistry and pharmacological effects: BioMed research international. BioMed Research International. 10(1155) : 925631.

Zoubi YE, Fouad EL, Farah A, Taghzouti K, Lalami AEO. 2017. Chemical composition and larvicidal activity of Moroccan Atlas Cedar (Cedrus atlantica Manetti) against Culex pipiens (Diptera: Culicidae). Journal of Applied Pharmaceutical Science 7(07):030-034.

Zu ZY, Jiang MD, Xu PP, Chen W, Ni QQ, Lu GM, Zhang LJ. 2020. Coronavirus disease 2019 (COVID19): a perspective from China. Radiology, 200490 